\section{RUN: A logistic-based interactive program to detect patterns of test item bias}

\author{
MICHEL G. BOUGON \\ Pennsylvania State University \\ University Park, Pennsylvania 16802
}

and

\author{
ROBIN I. LISSAK \\ University of Mlinois, Champaign, Illinois 61820
}

Statistical procedures for detecting item bias have received much attention in the last decade. Of these methods, the three-parameter logistic model (3PLM) has been demonstrated to be successful at detecting bias (Ironson \& Subkoviak, 1979; Rudner, Getson, \& Knight, 1980), as well as theoretically sound (Lord, 1980). ${ }^{1}$ In this model, each item is described by an item characteristic curve (ICC) that describes the probability $\mathrm{P}(\hat{\theta})$ of getting an item correct given ability, $\hat{\theta}$. The ICC is defined by three estimated parameters: discrimination $(\hat{a})$, difficulty $(\hat{b})$, and guessing $(\hat{c})$.

Despite the theoretical and empirical evidence supporting the use of 3PLM in detecting item bias, the model has not gained widespread use among practitioners. Ironson (Note 1) outlines some of the major reasons as (1) the requirement of large sample sizes and item pools by the parameter estimation program, LOGIST, (2) the cost of parameter estimation by LOGIST (and reestimation of parameters to detect item bias), (3) the poor estimation of the a and c parameters, and (4) the lack of test-of-fit statistics to determine how well the data fit the model. These problems have been addressed by Bougon and Lissak (Note 2), who introduced a procedure to compare items for bias using the 3PLM. The procedure involves the comparison of empirical characteristic curves (ECCs) instead of ICCs. ECCs are plots of the actual probabilities of a group of examinees' getting the item correct given $(\hat{\theta})$ ability. Thus, parameter estimation problems are avoided. The procedures of computing ECCs are described in detail in Bougon and Lissak (Note 2). The ECCs for each subgroup are then compared using the B statistic (Bougon \& Lissak, Note 2).

\section{The B Statistic}

The B statistic may be used to determine whether the ECC for one subgroup of examinees is different from the ECC for a reference group of examinees. For each point of the ECC, one computes the statistic,

$$
\mathrm{t}_{\mathrm{i}}=\left\{\frac{\tilde{\mathrm{p}}_{2}-\tilde{\mathrm{p}}_{1}}{\left[\left(\mathrm{n}_{2}-1\right)^{-1} \tilde{\mathrm{p}}_{2} \tilde{\mathrm{q}}_{2}+\left(\mathrm{n}_{1}-1\right)^{-1} \tilde{\mathrm{p}}_{1} \tilde{\mathrm{q}}_{1}\right]^{1 / 2}}\right\}_{\theta_{\mathrm{i}}}=\frac{\tilde{\mathrm{d}}_{\mathrm{i}}}{\tilde{\mathrm{s}}_{\mathrm{d}_{\mathrm{i}}}},
$$

where subscripts 1 and 2 refer to the first and second ECC, $\tilde{\mathbf{p}}$ indicates an observed examinee's probability of success, and $n$ is the number of examinees aggregated in the ECC point. Then, one computes the A statistic,

$$
\mathbf{A}=\sum_{\mathbf{K}} t_{\mathbf{i}}
$$

where $\mathrm{K}$ is the number of ECC points over which the ratios $t$ are computed. Next, one computes the B statistic,

$$
\mathrm{B}=\frac{\nu^{1 / 2}}{\mathrm{~K}} \mathrm{~A}
$$

where $v$ represents the degree of freedom associated with the sum of ts.

In the case of two ECCs, the B statistic reduces to

$$
\mathrm{B}=\frac{1}{\mathrm{~K}^{1 / 2}} \mathrm{~A}=\frac{1}{\mathrm{~K}^{1 / 2}} \Sigma \mathrm{t}_{\mathrm{i}}
$$

The B statistic is asymptotically Gauss distributed and nearly Gauss distributed when each point of the ECC contains about $\mathbf{4 0}$ or more examinees (Bougon \& Lissak, Note 2).

\section{The APL Function}

RUN is an interactive program written in standard APL that will run on any computer having APL in its system library. In typical applications, a $16 \mathrm{~K}$ - to $32 \mathrm{~K}$ byte work space is sufficient to store the input and output data matrices, the functions called by RUN, and to provide the work space necessary for the functions to execute.

RUN is designed for a user who wants to investigate bias, item by item, in a test. Since RUN is interactive, provides clear user prompts, and performs multiple checks of user responses, little computer experience is needed for its use. RUN queries the user for the type of information and outputs that he/she wants and for the data and information that it needs to conduct the analyses.

Once RUN has been invoked interactively at a terminal, RUN queries the user to determine (1) whether to run the standard B statistic (or experimental analyses under investigation by the authors) and (2) whether to display a graph of the two ECCs for the item being analyzed. If a graph is displayed, the corresponding plotting vectors are stored for reexamination or replotting on a hard-copy terminal.

Input. RUN asks the user whether he/she wants to (1) compare ECCs (or use other experimental comparisons) and (2) display and store the graphs of the ECCs 
for each item. RUN then asks for ( 3 and 4 ) the names of the two matrices of logistic parameters, (5 and 6) the names of the two probability matrices, $(7$ and 8$)$ the two vectors of examinees' thetas, $(9$ and 10$)$ the number of points in each ECC, and (11) a list of which items in the test are to be tested for bias. Finally, RUN asks the user (12) whether he/she wants to run automatically (no new data have to be entered and all specified items will be analyzed) or manually (the user must specify which item he/she wants to analyze individually but does not have to reenter the basic data). RUN also allows the user (13) to stop a RUN in progress, while at the same time saving the outputs of the analyses already performed.

Functions Performed by RUN. RUN checks both the user and data inputs, prepares and names the output matrices, and prepares the data needed by the functions that it calls. It also calculates a common theta metric for the ECCs, calculates and executes the correction aligning the ECCs, and generates the interactive outputs. It then computes the B statistic for each item and stores the interactive outputs.

Interactive Output. Under user's control, RUN displays the value of the B statistic (as well as some other experimental statistics) for each item analyzed and plots the item's ECCs. If an added option is selected by the user, the vectors of thetas, differences, and $t$ ratios of the ECCs are displayed. Each successive interactive output remains displayed on the CRT until the user enters a cursor return. This applies to hard-copy terminals as well.

Summary Output. All the interactive outputs are stored in matrices for further examination and analysis. These matrices are named automatically by RUN (after the names of the input matrices), thus alleviating any subsequent doubt about the origin of these outputs when many tests are examined. A function called MSV may be used to check the distribution of the B statistic for any analysis. A REPLOT function allows the user to reexamine any of the ECC plots presented during RUN's execution, as well as to obtain a hard copy if the RUN analyses were done on a CRT.

\section{Summary}

RUN is designed to provide an investigator who has little computer experience information about patterns of bias between items and within items. The program is designed to be used on any standard version of APL. Summary outputs may be easily transferred to non-APL systems for further analysis if needed. Cost-saving features such as automatic RUN and graph plotting have been included. The graph-plotting features of RUN rely on the input data needed for standard RUN analyses and are automatically supplied by RUN if the option is selected. This avoids the need for complex and costly plotting subroutines.

\section{Availability}

A source copy of the program, sample input, and sample output are available from the authors without charge. Michel Bougon is presently at the Department of Organizational Behavior, Pennsylvania State University, 609 Business Administration Building, University Park, Pennsylvania 16802.

\section{REFERENCE NOTES}

1. Ironson, G. Chi-square and latent trait approaches to the measurement of item bias. Paper presented at the Third Annual Johns Hopkins University NSER: "Item Bias: The State of the Art," Washington, D.C., November 1980.

2. Bougon, M. G., \& Lissak, R. I. Detecting item bias using the 3-parameter logistic model and the B-statistic. Manuscript submitted for publication, 1981.

3. Warm, T. A. A primer of item response theory (Tech. Rep. 941078). Oklahoma City: U.S. Coast Guard Institute, Department of Transportation, 1978.

\section{REFERENCES}

Ironson, G. H., \& Subkoviak, M. J. A comparison of several methods of assessing bias. Journal of Educational Measurement, 1979, 16, 209-225.

LORD, F. M. Application of item response theory to practical testing problems. Hillsdale, N.J: Erlbaum, 1980.

Rudne R, L. M., Getson, P. R., \& Knight, D. C. A Monte Carlo comparison of seven biased item detection techniques. Journal of Educational Measurement, 1980, 17, 1-10.

\section{NOTE}

1. Readers not familiar with latent trait models are referred to Warm (Note 3).

(Accepted for publication September 6, 1981.) 